\title{
SLAVE ANALOGWORK IN DER BRASILIANISCHEN TEXTILINDUSTRIE
}

\section{ORIGINALER ARTIKEL}

NETO, Dalk Dias Salomão ${ }^{1}$, SOUSA, Nicole Moreira Faria², DENDASCK, Carla Viana $^{3}$, FECURY, Amanda Alves ${ }^{4}$, OLIVEIRA, Euzébio de ${ }^{5}$, DIAS, Claudio Alberto Gellis de Mattos $^{6}$

NETO, Dalk Dias Salomão. Et al. Slave analogwork in der brasilianischen textilindustrie. Revista Científica Multidisciplinar Núcleo do Conhecimento. Jahrgang 06, Ed. 05, Vol. 13, S. 28-46. Mai 2021. ISSN: 2448-0959, Zugriffsverbindung: https://www.nucleodoconhecimento.com.br/sozialwissenschaften/brasilianischentextilindustrie, DOI:

10.32749/nucleodoconhecimento.com.br/sozialwissenschaften/brasilianischentextilindustrie

\footnotetext{
${ }^{1}$ Rechtsanwalt, Bachelor of Law (CEAP - Center for Higher Education of Amapá), Spezialist für Arbeitsrecht und Arbeitsprozess der Bildungseinrichtung Damásio.

${ }^{2}$ Rechtsanwalt, Bachelor of Law (CEAP - Center for Higher Education of Amapá), Spezialist für Zivilprozessrecht der Institution Damásio Educacional.

${ }^{3}$ Theologe, PhD in Clinical Psychoanalysis. Er arbeitet seit 15 Jahren mit Scientific Methodology (Research Method) in der Beratung der wissenschaftlichen Produktion von Master- und Doktoranden. Der Spezialist für Marktforschung und -forschung konzentrierte sich auf Gesundheit.

${ }^{4}$ Biomedizin, PhD in Tropenkrankheiten, Professor und Forscher des Medical Course of Macapa Campus, Federal University of Amapa (UNIFAP), Pro-Rektor of Research and Graduate Studies (PROPESPG) der Föderalen Universität von Amapá (UNIFAP).

${ }^{5}$ Biologe, PhD in Tropenkrankheiten, Professor und Forscher des Physical Education Course, Federal University of Pará (UFPA).

${ }^{6}$ Biologe, PhD in Theorie- und Verhaltensforschung, Professor und Forscher des Chemie-Studiengangs des Instituts für Grund-, Technik- und Technologiepädagogik (IFAP) und des Graduate Program in Professional and Technological Education (PROFEPT IFAP).
}

RC: 86627

Zugriffsverbindung: https://www.nucleodoconhecimento.com.br/sozialwissenschaften/brasilianischentextilindustrie 


\section{ZUSAMMENFASSUNG}

Das Institut der Sklaverei ist in der Menschheit seit dem Beginn der Existenz des Menschen präsent. Die Sklaverei in Brasilien hat die Wirtschaft seit Jahrhunderten aufrechterhalten. Millionen von Afrikanern wurden aus ihrer Heimat geholt und in erniedrigende Lebens- und Arbeitsbedingungen versetzt. Der Prozess der Abschaffung der Sklaverei war zeitaufwändig und schrittweise. Es gab Jahrhunderte des Kampfes und des Leidens, damit die Welt begann, das Böse zu erkennen, das die Sklaverei darstellt. Selbst nach der Abschaffung der Sklaverei war es üblich, den Arbeiter im Feld durch Schulden oder durch Gesetze gefangen zu sehen, die Arbeitgeber in Bezug auf den Arbeitnehmer ermächtigten. Ziel dieser Forschung war es, die Arbeitsbedingungen analog zum Sklaven in der brasilianischen Textilindustrie zu analysieren. Es wurde mit bibliographischer Überprüfung und qualitativer Analyse durchgeführt. Durch seine neue Kleidung wurde zeitgenössische Sklavenarbeit für einige Zeit unsichtbar. Die Faktoren, die es ermöglichen, dieses Verbrechen zu begehen, auch wenn es heute im Wesentlichen mit einem Stativ zusammenhängt: Straflosigkeit, Armut und Profit. Die Situation des Elends der bedürftigsten Bevölkerung zwingt sie, sich unter untermenschlichen Bedingungen einer Arbeit zu unterziehen. Bei diesen Textilarbeitern handelt es sich hauptsächlich um Einwanderer aus benachbarten, unterentwickelten Ländern aus Lateinamerika. Brasilien war eines der ersten Länder der Welt, das diese Art von Arbeit anerkannte, und dass gemeinsam mit der Internationalen Arbeitsorganisation (OIT) und austernden Nichtregierungsorganisationen versuchen, diese kriminelle Praxis auf ihrem Territorium zu bekämpfen.

Schlüsselwörter: Sklaverei, Gesetzgebung, Kampf, Industrie.

\section{EINLEITUNG}

Das Institut der Sklaverei ist in der Menschheit seit dem Beginn der Existenz des Menschen präsent. Obwohl sie im Laufe der Geschichte unterschiedliche 
Bedeutungen, Formen und Ziele präsentiert hat, war die Sklaverei immer von der Herrschaft des jeweils anderen geprägt (Mota e Ramos, 1999)

Für Oliveira (2011) begann die Beziehung zwischen Männern in der prähistorischen Phase, aufgrund der prekären Lebensbedingungen und der Notwendigkeit für Jagd, Fischerei und Obstsammlung. Und es war durch den Austausch von Erfahrungen und die Zusammenarbeit zwischen den Individuen, dass die ersten Stämme entstanden.

Das Institut der Sklaverei begleitet den Menschen vom Beginn der Menschheit an, wie bereits gesagt wurde, mit Beweisen für seine Existenz in verschiedenen Momenten der Menschheit und in unzähligen Formen. In der Heiligen Bibel (dem Hauptbuch der Christen) finden wir beispielsweise zahlreiche Fälle von Sklaverei, die in den Evangelien zitiert werden. Die Sklaverei beruhte damals auf der Knechtschaft durch Schulden oder Arbeit, die sich aus der Unterwerfung des Verlierers durch den Gewinner unter anderem ergab (Oliveira, 2011).

Mit dem Einsatz von Sklaverei für schwerere Jobs haben es Menschen wie die Griechen geschafft, Philosophie und Kunst zu entwickeln wie kein anderer. Gleichzeitig mit der kommerziellen Produktion gab es eine große Ausweitung der handwerklichen und landwirtschaftlichen Produktion, die durch den Export- und Importhandel kanalisiert wurde (Oliveira, 2011).

Für Silva (2010), wie in Griechenland, Rom verwendet Sklavenarbeit, und es war während der Zeit des Reiches, dass die Sklaverei ihren Höhepunkt erreichte und erreichte insgesamt 30\% der römischen Gesellschaft.

Es gab viele Möglichkeiten, in Rom Sklave zu werden, in der Regel war jeder Sohn einer Sklavenmutter auch Ein Sklave. Eine andere Möglichkeit, jemanden zu versklaven, war durch Krieg, mit Gefangenen, die zur Zwangsarbeit gezwungen wurden, Sklaverei wurde auch als eine Möglichkeit benutzt, Einzelpersonen zu bestrafen, wie im Falle der Desertion der Armee oder der Schuldhaft von Schulden (Silva, 2010). 
Die Gesellschaft des Mittelalters wurde von Feudalherren, Geistlichen und Dienern gegründet. In dieser Zeit war die Sklaverei nicht das wichtigste Mittel der Arbeit, da sie die Ersatzdiener der Sklaven war und schreckliche Lebens- und Arbeitsbedingungen erlitt (Silva, 2010).

Obwohl die Diener nicht als Objekte betrachtet wurden, war ihre Rechtslage nicht so anders als die der Sklaven, da sie als bloße Steile des Landes behandelt wurden, unter Auflagen einer persönlichen Anordnung, ohne ihr Recht zu kommen und zu gehen garantiert und sogar ein Verbot der Ehe ohne Genehmigung (Silva, 2010).

Es ist wichtig, den Fokus auf unser Land zu legen, um darauf hinzuweisen, dass die Sklaverei in der historischen Entwicklung dieses großen Teils des Landes vorhanden war. Ursprünglich bekannt als Santa Cruz de Cabrélia und später aus Brasilien, wurde es von Portugiesen kolonisiert, die, als sie hier im Jahr 1500 ankamen, großangelegte Sklaverei mitbrachten, beginnend mit den Eingeborenen, Tupis und Guaranes hauptsächlich und später Schwarzafrikaner (Silva, 2010).

Um bei der Suche nach Rohstoffen für die Metropole erfolgreich zu sein, begannen die Portugiesen mit der Kolonisierung unter Verwendung der Sklaverei als Extraktionsbasis. Zuerst benutzten sie die einheimische Arbeit und machten den Tauschhandel mit ihnen, die im Austausch gegen Gewürze und Metalle den Indianern Objekte von irrelevantem Wert wie Spiegel und Kämme überließen (Oliveira, 2011).

Das Verhältnis der portugiesischen Krone und der Eingeborenen war zunächst recht friedlich, aber nachdem die Portugiesen beschlossen hatten, das Gebiet zu besetzen, um wirtschaftliche Ausbeutung zu entwickeln, änderten sich die Beziehungen. Von da an begannen die Siedler, die Eingeborenen aus ihrem Land zu vertreiben und sie der Sklavenarbeit zu unterwerfen, die unter physischer Ausbeutung und neuen Krankheiten litt, die der weiße Mann mit sich brachte (Gorender, 1985).

Indigene Völker litten lange Zeit unter der Sklaverei, aber diese Situation dauerte nicht lange an, angesichts mehrerer Faktoren, die die Ausbeutung der Einheimischen 
verlangsamten, wie die geringe Bevölkerungsdichte der indigenen Völker; die Stämme, die unamalike wurden, als sie ihre Versklavung wahrnahmen; die indigene Bevölkerung, die schließlich aufgrund von Ausbeutung und Krankheiten dezimiert wurde, die bisher unbekannt waren, sowie der Schutz, den die Jesuiten erhielten (Campos, 2015).

Der gleiche Schutz, den die Indigenen von den Jesuiten gewährten, wurde den Schwarzen nicht gewährt, so dass ihre Versklavung praktisch ein Konsens zwischen Kirche und Krone war (Fausto, 2004). Eine der Hauptgründe für die Versklavung des afrikanischen Schwarzen war, dass diese Praxis bereits in Afrika üblich war, auch auf der Grundlage wissenschaftlicher Theorien, die die Minderwertigkeit der schwarzen Rasse bestätigten, weil Menschen mit geringer Intelligenz und emotional instabiler, biologisch zur Unterwertkeit bestimmt, demonstriert wurden, wodurch eine der größten Formen von Vorurteilen entstand, die je gesehen wurden (Mattos, 2015).

Die Sklaverei in Brasilien hat die Wirtschaft seit Jahrhunderten aufrechterhalten. Millionen von Afrikanern wurden aus ihrer Heimat geholt und in erniedrigende Lebensund Arbeitsbedingungen versetzt. Nach (Soares, 1860) kamen etwa 371.615 Sklaven indas Land.

Anfangs konzentrierten sich Sklavenarbeit und die brasilianische Wirtschaft auf dem Feld, in der Landwirtschaft, genauer gesagt in Zuckerrohrplantagen im Nordosten, als ein Weg, der von der Krone der Kolonisierung dieses Teils der "neuen Welt" gefunden wurde. Es ist wichtig zu zeigen, dass im achtzehnten Jahrhundert, mit der fortschreitenden Ausdehnung der Kolonisierung und Entdeckung neuer Räume im Landesinneren, das große Bergbaupotenzial der Länder entdeckt wurde, wodurch ein intensiver Markt für die Gewinnung von Erten wie Gold geschaffen wurde, wo Sklavenarbeit solche Aktivitäten ermöglichte (Campos, 2015).

Und in diesem Tempo hat die Sklaverei nicht nur die Welt, sondern auch Brasilien negativ geprägt. Die einzige Rechtfertigung für die Einfuhr des Negers war das Werk. Sie arbeiteten Stunden am Ende, fünfzehn bis achtzehn Stunden am Tag, erlitten RC: 86627

Zugriffsverbindung: https://www.nucleodoconhecimento.com.br/sozialwissenschaften/brasilianischentextilindustrie 
täglich körperliche Gewalt und Psychologie und wurden als Objekte behandelt (Pinsky, 1992).

Der Prozess der Abschaffung der Sklaverei war zeitaufwändig und schrittweise. Es gab Jahrhunderte des Kampfes und des Leidens, damit die Welt begann, das Böse zu erkennen, das die Sklaverei darstellt. Es gibt mehrere wichtige Momente, die diesen Gedankenwechsel repräsentieren: die Ausrufung der Unabhängigkeit der Vereinigten Staaten, die auf der Erklärung der Menschenrechte beruhte; die Französische Revolution von 1789, die die Grundsätze der Freiheit, Gleichheit und Brüderlichkeit hervorhob. Erwähnenswert ist auch die englische Revolution, die mit dem Aufkommen der Maschine zeigte, daß die Produktion sogar mit freier Arbeit zunehmen konnte (Montenegro, 1997).

Vor diesem Grund mussten Händler und Produzenten nach einem Weg suchen, Sklavenarbeit zu ersetzen. In Kaffeeplantagen wurde beispielsweise Sklavenarbeit durch europäische Einwanderer ersetzt, im sogenannten Siedlungssystem (Stimulus des brasilianischen Staates), das gegen Entgelt arbeitete, der in dem Prozentsatz der Kaffeeproduktion festgelegt ist (Silva, 2010).

Selbst nach der Abschaffung der Sklaverei war es üblich, den Arbeiter auf dem Feld durch Schulden oder durch Gesetze gefangen zu sehen, die Arbeitgeber in Bezug auf den Arbeitnehmer durch vertragliche Verpflichtungen ermächtigten, wie harte Strafen, wie die Verhaftung des Arbeiters, der ohne fairen Grund vom Hof weg war oder der, auf dem Grundstück, sich weigerte zu arbeiten (Silva, 2010).

So versteht man, dass die Abschaffung der Sklaverei in Brasilien durch das AureaGesetz die Sklaven nicht wirklich befreite, weil es eine extrem rassistische und vorurteilsbehaftete Gesellschaft gab, was zu aktuellen Praktiken führte, die der Sklaverei analog entsprechen. 


\section{ZIEL}

Analyse der Arbeitsbedingungen analog zum Sklaven in der brasilianischen Textilindustrie.

\section{METHODE}

Die Forschung wurde mit bibliographischer Überprüfung und qualitativer Analyse durchgeführt.

Nach Lima und Mioto (2007): "[...]ein Forschungsprozess zu konstruieren, der sich auf die Definition der methodischen Verfahren bezieht, die diesen Prozess leiten werden, basiert auf der Beobachtung, die mehrere Forschungsberichte berichten".

Qualitative Forschung arbeitet mit Werten, Einstellungen und dem Verhältnis zwischen Prozessen und Phänomenen, nicht numerisch gemessen (Gerhardt und Silveira, 2009).

\section{ERGEBNISSE UND DISKUSSION}

\section{SKLAVENÄHNLICHE ARBEIT}

Die Existenz arbeitsbedingungen analog zum sklaven lässt sich durch die einfache Tatsache erklären, das alte Sklavensystem nicht zu überwinden, aufgrund seiner kulturellen Wurzeln im Laufe der Jahrhunderte, auch nach seinem Verbot. Darüber hinaus basiert die moderne Sklaverei auf einem Stativ: Straflosigkeit, Gier und Armut, die notwendig werden, nicht nur, um dieses Verbrechen wie jedes andere zu bekämpfen, sondern auch, um unser Justizsystem, unsere Konsummuster und Entwicklungsmodelle zu überprüfen (Miranda e Oliveira, 2010).

Daher ist es der Welt und ihren Bemühungen noch nicht gelungen, Sklavenarbeit auszulöschen und sich als mühsame Aufgabe zu erweisen, insbesondere für die Kultur und den Mangel an Mitgefühl unter den Menschen. Auf diese Weise sollten sowohl die RC: 86627

Zugriffsverbindung: https://www.nucleodoconhecimento.com.br/sozialwissenschaften/brasilianischentextilindustrie 
Staaten als auch die Gesellschaft im Allgemeinen bei dieser Art von Unsinn wachsam sein.

\section{SCHUTZPRINZIPIEN DER ARBEIT}

Rechtsgrundsätze sind definiert als eine Reihe von Verhaltensnormen, die im Rechtssystem dargestellt werden. Die Grundsätze und auch die Regeln sind Normen. Es kann bestätigt werden, dass alle Wissenschaft auf Prinzipien beruht, so dass das Gesetz dieser Regel nicht entgeht (Saraiva, 2012).

In Martins' Denken (2011, S. 62) bilden die Prinzipien die Säulen des Rechts, seine Grundlage, und die Prinzipien haben einen viel höheren Grad an Abstraktion als die Norm, und ihre Anwendung ist auf konkrete Fälle (Martins, 2011).

Die Prinzipien werden durch zwei Strömungen erklärt, jusnaturalist und legal positivismus, von denen die erste die Prinzipien über positives Recht stellt, die daher unter den Gesetzen vorherrschen. Für den zweiten würden die Prinzipien Integratoren des Gesetzes sein, die Lücken darin füllen (Nascimento, 2010).

Nicht durch die Entweichen vor der Regel wird das Arbeitsrecht durch eine Reihe von Grundsätzen und Regeln gebildet, die darauf abzielen, bessere Arbeitsbedingungen für die Arbeitnehmer durch Schutz zu gewährleisten.

Es ist wichtig, einen Grundsatz zu erwähnen, der, auch wenn er nicht im Arbeitsbereich ist, für das Rechtssystem als Ganzes von größter Bedeutung ist, das Prinzip der Würde des Menschen. Dieser Grundsatz hat einen moralischen und spirituellen Wert, der der Person innewohnt, daher wird jede Person mit diesem Gebot unterstützt, und dies stellt das maximale Prinzip des demokratischen Rechtsstaates dar.

Der Schutzgrundsatz ist das breiteste und wichtigste im Arbeitsrecht. Dieses Prinzip ist ein Grundsatz, der das Arbeitsrecht leitet, das darauf abzielt, den schwächsten Teil des Rechtsverhältnisses zu schützen, den Arbeitnehmer, der sich gegenüber dem Arbeitgeber ungeschützt sieht. $\mathrm{Er}$ versucht, mehr Bedingungen für RC: 86627

Zugriffsverbindung: https://www.nucleodoconhecimento.com.br/sozialwissenschaften/brasilianischentextilindustrie 
teilhypoausreichende Beziehung, der Mitarbeiter zu geben. Daher sieht sie die Schaffung von Mechanismen zur Verringerung der Ungleichheit zwischen den Parteien, zur Verhinderung der Ausbeutung von Arbeit und zur Gewährleistung des sozialen Wohlergehens der Arbeitnehmer vor (Saraiva, 2012).

Dieses Prinzip ist in der Regel in drei unterteilt: die "in dubio pro operário", die der Anwendung der günstigsten Standard und die der Anwendung der vorteilhaftesten Bedingung. Das Prinzip "in dubio pro operário" besagt, dass man angesichts von zwei oder mehr tragfähigen Auslegungen die günstigste für den Arbeitnehmer wählen muss, vorausgesetzt, dass es nicht der klaren Manifestation des Gesetzgebers widerspricht, noch ist es eine unerschwingliche Angelegenheit. Der Grundsatz der günstigsten Regel besagt, dass man sich im Falle eines Konflikts zwischen zwei oder mehr in diesem Fall geltenden Vorschriften für diejenige entscheiden sollte, die für den Arbeitnehmer vorteilhafter ist. Schließlich bestimmt das Prinzip der vorteilhaftesten Bedingung die Dauerhaftigkeit vorteilhafterer Bedingungen für den Arbeitnehmer, auch wenn es eine zwingende gesetzliche Regelung gibt, die das Gegenteil vorschreibt (Saraiva, 2012; Mattos, 2015).

Arbeitsrechte sind in der Regel für den Arbeitnehmer unverzichtbar. Dieser Grundsatz ist äußerst wichtig für den Schutz der Hypoausreichend, denn oft täuscht oder stärkt der Arbeitgeber durch Zwang den Arbeitnehmer, sich gegen seinen Willen zu entscheiden, indem er bereits erworbene Rechte aufgibt (Rodriguez, 2015). Das heißt, in der Regel kann der Arbeitnehmer nicht auf Das Arbeitnehmerrecht verzichten, zum Beispiel kann man den Urlaub nicht aufgeben, wenn dies geschieht, wird eine solche Handlung als null betrachtet (Martinez, 2015).

Das Prinzip des Primats der Wirklichkeit führt dazu, dass arbeitsrechtliche Beziehungen durch die Tatsachenlage definiert werden, d.h. durch die Art und Weise, wie die Dienstleistungen erbracht wurden, unabhängig von dem Namen, der ihnen von den Parteien zugewiesen wurde (Saraiva, 2012). 
Ein klares Beispiel ist, wenn Zeugenaussagen die Nichtbereitstellung einzelner Arbeitsmittel (PSA) nachgewiesen werden, obwohl es eine Unterschrift von Warnhinweise für Lieferungen gibt. Daher ist es klar, dass man die Wahrheit der Tatsachen durch Zeugen beweisen kann, zum Beispiel, indem man solche Beweise dem schriftlichen Dokument vorherrscht, wenn die Echtheit des Zeugnisses nachgewiesen wird.

\section{ALLGEMEINE ARBEITSBEDINGUNGEN ANALOG ZU SKLAVEN}

Früher wurden Sklaven als Objekte gesehen und heute nicht mehr. Obwohl sie derzeit als Personen angesehen werden, die mit Persönlichkeit unterstützt werden, sind sie immer noch erniedrigenden Lebens- und Arbeitsbedingungen unterworfungen (Mattos, 2015).

Bemerkenswert ist, dass es "arbeitsbedingungen analog zum sklaven" genannt wird, da die Sklavenarbeit selbst ausgelöscht wurde. Trotz aller Bemühungen sind solche Sklavereipraktiken jedoch nicht vollständig verschwunden.

Die Existenz sklavenähnlicher Arbeit kann durch die einfache Tatsache erklärt werden, das alte Sklavensystem trotz der Umsetzung der Lohnarbeit nicht zu überwinden, aufgrund seiner kulturellen Verwurzelung im Laufe der Jahrhunderte. Darüber hinaus basiert die moderne Sklaverei auf einem Stativ: Straflosigkeit, Gier und Armut, was es notwendig macht, dieses Verbrechen nicht nur wie jedes andere zu bekämpfen, sondern auch unser Justizsystem, unsere Konsummuster und unser Entwicklungsmodell zu überprüfen (Miranda und Oliveira, 2010).

Das Institut der Sklaverei hat derzeit eine neue Kleidung, mit mehreren Konfessionen für sklavenähnliche Arbeit: zeitgenössische Sklavenarbeit; Arbeit unter untermenschlichen Bedingungen, Schuldensklaverei, Zwangsarbeit, Überfischung der Arbeit, neue Sklaverei u.a., Zwangsarbeit, Erniedrigung der Arbeit unter anderem (Cristova und Goldschmidt, 2012). 
Die verschiedenen Bezeichnungen, die oben dargelegt sind, sind darauf zurückzuführen, dass es an Konsens über sein Konzept sowie an den Kriterien für seine Charakterisierung des Instituts mangelt. Aus diesem Grund gibt es eine Variation von Elementen in seiner Konzeptualisierung, sowie in den verwendeten Begriffen, um auf diese Art der Ausbeutung der Arbeiterarbeit zu verweisen (Cristova und Goldschmidt, 2012).

Mehrere Autoren haben sich bereits zu diesem Thema positioniert, und es gab kein einheitliches Verständnis zwischen den Gedanken. Es war jedoch in demselben Gefühl der Abscheu über diese unmenschlichen Esums deutlich, Menschen zu behandeln und den Arbeiter unter untermenschlichen Bedingungen zu unterwerfen (Brito Filho, 2004).

Die Realität des versklavten Arbeiters ist die von jemandem, der keine wahl, ohne Rechte. Solche Arbeitnehmer werden in der Regel an Orten weit weg vom Arbeitsplatz gelockt, ihnen wird ein guter Arbeitsplatz mit einem unterzeichneten Portfolio, einer großzügigen Vergütung und anderen Leistungen versprochen. Wenn der Arbeiter jedoch am Arbeitsplatz ankommt, ist er in wirklichkeit völlig anders als der versprochene, die meiste Zeit muss er die Kosten für Reisen, Wohnen und Essen tragen (Prado, 2005).

Der Begriff "Sklavenarbeit" erleichtert das Verständnis durch die Laienöffentlichkeit, da er Merkmale aufweist, die den Konzepten der Internationalen Arbeitsorganisation ähneln. Ein solches Konzept war sehr nahe an der "Zwangsarbeit" (Audi, 2006).

Angesichts dieser Konzepte können wir erkennen, dass Arbeit analog zum Sklaven existiert, oft aufgrund der Hyposuffizienz von Arbeitern, die auf der Suche nach dem Minimum für ihren Lebensunterhalt und ihre Familien am Ende ihre eigene Würde beiseite lassen und sich demütigender Arbeit unterwerfen (Campos, 2015).

Die Schwierigkeit, die Arbeit unter sklavenähnlichen Bedingungen nicht nur im akademischen Umfeld zu charakterisieren, betrifft öffentliche Agenten wie Richter, 
Staatsanwälte und Mitarbeiter von Arbeitspolizeistationen. Diese Schwierigkeit hat große Folgen, weil sie am Ende die Charakterisierung und Typisierung der Tat behindert, was es schwierig macht, das Verbrechen selbst wahrzunehmen.

Daher kann man auf der Grundlage der oben genannten Konzepte und Forschungen Arbeit unter Bedingungen, die dem Zustand des Sklaven ähnlich sind, als die Ausübung menschlicher Arbeit mit Einschränkung definieren, in jeder Form, die von der Freiheit des Arbeiters sein kann, ohne seine Mindestrechte zu haben, die Würde der menschlichen Person zu schützen.

Die offizielle Anerkennung sklavenähnlicher Arbeit in Brasilien fand 1995 statt, trotz mehrerer Beschwerden bei der OIT (Internationale Arbeitsorganisation) im Laufe der Jahre. Trotz dieses erschwerenden Faktors war Brasilien eines der ersten Länder der Welt, das international die Existenz zeitgenössischer Sklaverei übernahm (Miranda und Oliveira, 2010).

Wie das Ministerium für Arbeit und Beschäftigung (MTE) ausführte, ist sklavenähnliche Arbeit im Land sehr präsent, wie die eigenen Daten der MTE zeigen, aus denen hervorgeht, dass zwischen 1995 und 201036.759 Arbeiter aus sklavenähnlichen Bedingungen entfernt wurden (Mte, 2018).

Nach Angaben der Internationalen Arbeitsorganisation ist die Hauptursache der Sklaverei die wirtschaftliche Ausbeutung der Arbeiter, die schätzungsweise etwa acht Millionen Menschen unter diesen Bedingungen auf der Welt leben. In Brasilien arbeiten nach Angaben der brasilianischen Regierung etwa fünfundzwanzigtausend Menschen unter sklavenähnlichen Bedingungen. Für diesen Betrag befindet sich die höchste Konzentration in den nördlichen und zentralen Westlichen Staaten, und 90\% der Gesamtzahl bestehen aus Analphabeten, 90\% begannen mit der Ausbeutung von Kinderarbeit und 80\% haben nicht einmal eine Geburtsurkunde (Simón e Melo, 2007).

Brasilien, das von der OIT anerkannt wurde, war eines der ersten Länder, das sklavenähnliche Arbeit kannte und bekämpfte. So erfolgte seine Kriminalisierung durch 
Art. 149 des brasilianischen Strafgesetzbuches, das später durch das Gesetz Nr. 10,803/2003 (Brasilien, 1940; 2017) geändert wurde. Es ist sehr wichtig zu betonen, dass in Brasilien durch Artikel 149 der CP "sklavenähnliche Arbeit" eine Gattung ist, die andere Arten hat, wie zwangs- und erniedrigende Arbeit (Brasil, 2017).

Seit der Änderung von Artikel 149 des PCs durch das Gesetz Nr. 10,803 vom 11. Dezember 2003 hat der Kampf gegen sklavenähnliche Arbeit einen großen Fortschritt gemacht, weil es einfacher geworden ist, eine solche Straftat zu charakterisieren.

Arbeitsbedingungen analog zum sklaven ist eine Gattung, die einige Arten besitzt. Für einige Gelehrte in der Gegend variieren diese Arten und können Zwangsarbeit, erschöpfende Arbeit, erniedrigende Arbeit und Schulden sein.

Simón e Melo (2007) verwendet die Nomenklatur "Arbeit unter sklavenähnlichen Bedingungen" und ist in drei Arten unterteilt: Zwangsarbeit, Arbeit unter erniedrigenden Bedingungen und Schuldenknechtschaft. Solche illegalen Arbeitsformen hatten keine Rechtswirkungen, da sie null und nichtig sind, und der Kampf gegen diese Praktiken wird nach brasilianischem Recht strafrechtlich durchgeführt.

Der Gelehrte Greco (2008) stellt fest, dass Sklavenarbeit derzeit auftritt, wenn eine Person eine andere zwingt, Zwangsarbeit zu leisten, erschöpfende Reisen erfordert, den Arbeiter erniedrigenden Bedingungen unterworfen oder seine Fortbewegung aufgrund von Schuldverschreibungen beschränkt.

Silva (2010) zeigt auch, dass die Nötigkeit des Arbeiters, so dass er die Arbeit nicht verlässt, mehrere Naturen haben kann, moralische oder psychologische, wie es bei den Bedrohungen der geistigen Unversehrtheit des Arbeiters, auch des physischen, auftritt, und der Arbeiter kann den Arbeitsplatz nicht verlassen, weil er sonst körperliche Bestrafung erleiden wird, oft mit bewaffneter Überwachung.

Für Araújo Júnior (2006) ist die Arbeit unter erniedrigenden Bedingungen durch die Nichteinhaltung der Grundregeln für Sicherheit und Gesundheitsschutz bei der Arbeit durch den Arbeitgeber gekennzeichnet, der keine medizinischen Untersuchungen des RC: 86627

Zugriffsverbindung: https://www.nucleodoconhecimento.com.br/sozialwissenschaften/brasilianischentextilindustrie 
Arbeitnehmers durchführt und keine persönliche Schutzausrüstung (EPI) garantiert, noch ein Ort zum Schutz der Arbeitnehmer vor dem Wetter, zusätzlich zur Aufrechterhaltung der Unterkunft ohne die geringsten Hygienebedingungen und ohne ausreichende Nahrung.

Es gibt auch eine erschöpfende Arbeit, die als anstrengende Arbeit bekannt ist, bei der der Arbeitnehmer über die gesetzlich zulässigen Fristen hinaus auf Arbeitsbedingungen getestet wird, was dem Arbeitnehmer großen Schaden zufügen kann. Die erschöpfende Reise kann als eine, die über die Grenzen des Prinzips der Würde des Menschen hinausgeht, konzipiert werden. Eine solche anstrengende Reise bedeutet nicht nur die überhöhte Anzahl von Stunden, sondern auch das unzureichende Tempo (Campos, 2015).

Der Gelehrte Proner (2010) stellt fest, dass ein erschöpfender Arbeitstag den Arbeiter negativ beeinflusst, weil er inm Momente der Freizeit und Bildung, des sozialen und familiären Lebens nimmt, die zu psychischen und körperlichen Erkrankungen führen können, weil er anfällig für den Erwerb einer Berufskrankheit wird.

Laut Bales (2001) ist Schuldensklaverei eine moderne Form der Ausbeutung menschlicher Arbeit, die häufigste in der Welt und vor allem in Brasilien. Diese Bedingung tritt auf, wenn die Person verpflichtet ist, für eine andere aufgrund von Darlehen vertraglich gebunden zu arbeiten. Arbeitsverträge werden mit angeblichen Arbeitsgarantien angeboten, in der Regel an geografisch abgelegeneren Orten, auf Bauernhöfen oder Fabriken, aber wenn sie auf dem Gelände ankommen, ist die Realität eine andere, getäuschte Arbeiter werden am Ende versklavt, dienen dem Vertrag nur, um den Arbeiter zu täuschen und ihn zu Fehlern zu führen.

Laut Audi (2006) können wir schlussfolgern, dass trotz zahlreicher beschriebener Wege alle Formen der Arbeit analog zum Sklaven immer zwei Gemeinsamkeiten haben: die Anwendung von Zwang und die Verweigerung der Freiheit. 


\section{FAKTOREN, DIE ZU ARBEITSBEDINGUNGEN ANALOG ZUM SKLAVEN IN BRASILIEN BEITRAGEN}

1988 wurde das Aurea-Gesetz veröffentlicht, das die Sklaverei im Land abschaffte. Es war jedoch nicht effizient, sie auszurotten, aufgrund mehrerer sozialer Faktoren, wie der Disqualifikation der Arbeitskräfte der "Ex-Sklaven" (Cristova und Goldschmidt, 2012).

Der Autor Monteiro (2011) beobachtete wesentliche Faktoren für die Dauerhaftigkeit der Sklavenarbeit im Land und definierte sie in einem Stativ: Straflosigkeit, Armut und Profit.

Was den Gewinn betrifft, so bezieht er sich auf Begünstigte, die Sklavenarbeit als eine Möglichkeit nutzen, hohe Gewinne zu erzielen, leichter, da sie sich nicht verpflichtet fühlen, die Arbeitsgesetze einzuhalten (Monteiro, 2011).

Was die Armut betrifft, so ist sie die Hauptursache dafür, dass sich viele Arbeitnehmer der Arbeit unter sklavenähnlichen Bedingungen unterwerfen, da sie bereit sind, auch mit unmenschlichen Vorschlägen zuzustimmen, um aus der extremen Armut herauszukommen und ihre Familien zu unterstützen (Monteiro, 2011).

Nach Angaben der Internationalen Arbeitsorganisation ist die Hauptursache der Sklaverei die wirtschaftliche Ausbeutung. Und zusammen mit diesen Informationen kann die Globalisierung auf den Märkten der Hauptgrund sein, da sie großen Wettbewerb erzeugt, was Hersteller/Produzenten dazu veranlasst, dem System zuzustimmen und zu sehr niedrigen Kosten zu produzieren (Cristova und Goldschmidt, 2012).

In Bezug auf die vorherrschenden Faktoren für die Existenz von Sklavenarbeit in ländlichen Gebieten, erklärt Silva (2009), dass sie die arme Region der Landarbeiter beeinflussen, da sie ein Gebiet mit einer großen Anzahl von Arbeitslosen sind, die vom Auftragnehmer durch falsche Versprechungen überzeugt werden, ohne formellen 
Arbeitsvertrag oder einen Standard, der durch die Consolidation of Labor Laws (CLT) festgelegt wurde.

In Bezug auf das zuvor erläuterte Wissen, dass für Arbeitgeber günstige Gerichtsentscheidungen zur Aufrechterhaltung der Sklavenarbeit in Brasilien beitragen, ergänzt Silvas (2009) Meinung, dass es keine strenge Strafe in der Gesetzgebung gibt, um diejenigen zu bestrafen, die für die Ausbeutung sklavenähnlicher Arbeit verantwortlich sind.

Angesichts des Fehlens zufriedenstellender Rechtsvorschriften ist das erzeugte Gefühl straflos, was den Eindruck vermittelt, dass die Übeltäter mit demselben kriminellen Verhalten fortfahren können, da sie keine schwerwiegenden Folgen für ihr Handeln haben werden (Silva, 2009).

Die Ursachen der Sklavenarbeit im Land sind für den Autor Damio (2014) vor allem die schlechte Einkommensverteilung und die mangelhafte Bildung. Das Elend, das durch die ungerechte Einkommensverteilung entsteht, führt, wie bereits gesagt, dazu, dass die Arbeiter den unmenschlichen Estinien der Sklavenarbeit unterworfen werden. Auf der anderen Seite erleichtert unbefriedigende Bildung es den Arbeitnehmern, sich täuschen zu lassen, so dass sie nicht in der Lage sind, für ihre Arbeitsrechte zu kämpfen.

In den letzten Jahren wurden mehrere Kampfmechanismen geschaffen, zum Beispiel die "schmutzige Liste" und nationale Pläne zur Ausrottung sklavenähnlicher Arbeit. Trotz all dieser Geschichte des Kampfes und der Überwachung fand 2017 die Reform des Arbeitsrechts in Brasilien durch das Gesetz 13.467/17 statt, das einen Rückschlag in diesem Thema brachte, die Praxis der Kriminalität verharmloste und die Prävention behinderte (Costa, 2015). 


\section{FORMEN ZUR BEKÄMPFUNG UND ERADIKATION DER ZEITGENÖSSISCHEN SKLAVENARBEIT}

In Brasilien hat die Konfrontation mit diesem Verbrechen in den letzten Jahrzehnten an Stärke gewonnen, trotz der Verzögerung, die die jüngste Arbeitsreform erfahren hat. Es ist bekannt, dass es unzählige Fälle von Menschen gibt, die in Bedingungen leben, die der Sklaverei entsprechen und die prekäre Überwachung des Staates umgehen (Oliveira, 2011).

Der Kampf und die Ausrottung der zeitgenössischen Sklavenarbeit ist viel schwieriger, als sie scheinen, da die Situation nicht einfach dadurch gelöst wird, dass der Arbeiter aus der prekären Situation herausgenommen und die Täter bestraft werden. Was in der Tat getan werden muss, ist eine Änderung des kapitalistischen Entwicklungsmodells, die durch die Suche nach Profit das Leben ganzer Familien beendet (Miranda e Oliveira, 2010).

Es gibt mehrere Möglichkeiten, sklavenähnliche Arbeit zu bekämpfen, sowohl gerichtliche als auch außergerichtliche. Es ist jedoch die öffentliche Politik, die den Umerziehungs- und kulturwandel der Gesellschaft beeinflusst, die der wichtigste Weg ist, dieses soziale Labyrinth zu überwinden (Silva, 2010).

In Brasilien gibt es eine gemeinsame Zusammenarbeit mehrerer öffentlicher Stellen, um die Ausbeutung sklavenähnlicher Arbeit auszumerzen, nämlich: Nationales Institut für Kolonisierung und Agrarreform (INCRA); Bundespolizei (PF); Federal Highway Police (PRF); Ministerium für Arbeit und Beschäftigung (MTE) und Staatsanwaltschaft (MP). Es ist wichtig zu beachten, dass die isolierte Arbeit nur einer Institution nicht wirksam ist. Ein weiterer wichtiger Weg, sie zu bekämpfen, ist die Gesellschaft selbst, die durch anonyme Beschwerden schnellere Antworten auf die Lösung solcher Probleme liefern kann.

Im Gegensatz zur Praxis der zeitgenössischen Sklavenarbeit ist es wichtig, einige Autoren, wie das Öffentliche Ministerium für Arbeit und Arbeitsjustiz, das eine sehr RC: 86627

Zugriffsverbindung: https://www.nucleodoconhecimento.com.br/sozialwissenschaften/brasilianischentextilindustrie 
wichtige Rolle spielt, durch Repressionsmaßnahmen, nehmen als Beispiel die öffentlichen Zivilklagen für moralische Schäden, die finanzielle Wiedergutmachung anstrebt.

Es ist wichtig, die Schaffung des Nationalen Plans zur Beseitigung der Sklavenarbeit hervorzuheben, der vom Sonderausschuss des Rates zur Verteidigung der Rechte der menschlichen Person (CDDPH) ausgearbeitet wurde, der landesweit tätig ist. Dieser Plan bringt Einrichtungen und Behörden im Zusammenhang mit dem Thema zusammen, die die Schaffung und Aufrechterhaltung einer dauerhaften öffentlichen Politik anstreben, die von nationalen Stellen oder einem Forum überwacht wird, das sich der Unterdrückung der Sklavenarbeit widmet.

Eine andere Strategie zur Bekämpfung solcher Verbrechen in Brasilien stammt von der Nationalen Kommission für die Beseitigung der Sklavenarbeit (CONATRAE), die Initiativen artikuliert und durchführt. Laut Oliveira (2011) wurde dieses Gremium im August 2003 geschaffen und wird von einem Kollegium gebildet, das mit dem Sondersekretariat für Menschenrechte der Präsidentschaft der Republik verbunden ist und als Hauptaufgabe die Umsetzung des Nationalen Plans zur Beseitigung der Sklavenarbeit überwacht.

Eine andere Form des Zusammenstoßes ist der Nationale Pakt für die Ausrottung der Arbeit in Brasilien. Diese Initiative basiert auf internationalen Bemühungen durch den Einfluss der OIT (Internationale Arbeitsorganisation, ein ONU Gremium). Der oben erwähnte Pakt arbeitet mit der Zusammenarbeit staatlicher Inspektionsbehörden zusammen, die versuchen, zeitgenössische Sklavenarbeit zu lokalisieren und zu bestrafen. Laut Miranda und Oliveira (2010) haben diese Bemühungen Ergebnisse erzielt und unterstützungserhalten von Vertretern von Unternehmen, die zusammen mehr als $25 \%$ des nationalen PIB ausmachen.

Eine der Möglichkeiten, die Arbeit analog zum bekanntesten Sklaven Brasiliens zu bekämpfen, ist die "schmutzige Liste". Dieses Instrument funktioniert wie folgt: Es wurde ein Arbeitgeberregister geschaffen, das die Arbeitnehmer verpflichtet, sich RC: 86627

Zugriffsverbindung: https://www.nucleodoconhecimento.com.br/sozialwissenschaften/brasilianischentextilindustrie 
Bedingungen zu unterwerfen, die denen von Sklaven entsprechen. Diese Liste ist ein öffentlicher Transparenzmechanismus des brasilianischen Staates, der 2003 geschaffen wurde und versucht, die Namen von Personen oder juristischen Personen offenzulegen, die mit Sklavenarbeit erwischt wurden (Mattos, 2015).

Das vorgenannte Register wurde durch die Verordnung Nr. 1.234 des Ministeriums für Arbeit und Beschäftigung von 2003 geregelt, die später durch die interministerielle Verordnung Nr. 2 vom 12. Mai 2011 ersetzt wurde, ein in Kraft gefundenes Dokument (Campos, 2015). Es wird wahrgenommen, dass diese Form des Kampfes von großer Bedeutung im Kampf gegen die zeitgenössische Sklavenarbeit und die Liste der Arbeitgeber wird, die von der ONU betrachtet werden, ein Referenzmodell in der Welt.

Im gleichen Sinne des Gesetzes Nr. 14.946/2013 wurde das Gesetz Nr. 14.946/2013 vom Staat Sao Paulo geschaffen, der darauf abzielt, die Aktivitäten von Unternehmen, die in ihrer Produktionskette den Einsatz von Arbeitnehmern unter Bedingungen analog zur Sklaverei haben, zu suchen und zu verbieten. Dieses Gesetz stammt aus einem Gesetzentwurf Nr. 1.034 von 2011 von dem Kongressabgeordneten Carlos Bezerra Junior, mit einer Besonderheit, weil es auf die Realität der Arbeiter in der Textilindustrie von Sao Paulo gerichtet ist (Mattos, 2015).

Die in diesem Gesetz zum Ausdruck gebrachte Form des Kampfes beruht auf der Löschung der staatlichen Steuerpflichtigkeit der Steuer auf den Waren- und Dienstleistungsverkehr (ICMS). So hat der Arbeitgeber, der direkt oder indirekt von sklavenähnlicher Arbeit profitiert, seine Eintragung widerrufen, zusätzlich zu den dem Gegenstand entsprechenden Rechtsvorschriften beschränkt, so wird eine solche Bestrafung in Art. 1 dieses Gesetzes (Dou, 2013) dargestellt.

Art. 4 des Gesetzes Nr. 14,946 des 2013 des Staates Sao Paulo, sobald die iCMSRegistrierung widerrufen wurde, ist die rechtsverletzende juristische Person nicht in der Lage, Tätigkeiten in der gleichen Branche durchzuführen, auch an einem anderen Ort, und die Partner sind daran gehindert, andere Gesellschaften der gleichen Tätigkeit 
für einen Zeitraum von 10 Jahren, ab dem Zeitpunkt der Amtsenthebung (Campos , 2015).

Die Sklavenarbeit ist zwar seit Hunderten von Jahren abgeschafft, aber im Widerspruch zu den Menschenrechten in der Gesellschaft präsent. Die gegenwärtige Sklaverei beruhte, anders als früher, nicht mehr auf dem Eigentum des Herrn über Sklaven, geschweige denn auf dem Geschäft des Kaufs und Verkaufs von Arbeitern, sondern auf der übermäßigen Kontrolle des Unternehmers über den Arbeiter, mit Mitteln wie Zwang und Zwang, mit dem Ziel, seine Profite zu steigern.

Einer der Wirtschaftszweige, in denen eine solche kriminelle Praxis sehr präsent ist, ist die Textilindustrie. In den großen Hauptstädten der Welt gibt es Sklavenarbeit nicht mehr überwiegend ländlich und präsentiert sich als urban, vor allem in Nähfabriken.

In der Realität der städtischen Sklavenarbeit verlassen viele Menschen ihre Häuser, verlassen Familien auf der Suche nach besseren Lebensbedingungen und unterwerfen sich am Ende Praktiken, die der Sklaverei, hauptsächlich aus DerNot, in großen Textilfabriken entsprechen (Campos, 2015).

Daher ist die Textilindustrie einer der großen Entdecker der zeitgenössischen Sklavenarbeit. Laut Mattos (2015) profitiert die Textilindustrie direkt von zeitgenössischer Sklavenarbeit, vor allem, weil ihre Produktion kurzfristig ist und China einer der Hauptnutzer dieser Art von Arbeit ist, was sie auf diesem Markt extrem wettbewerbsfähig macht.

Mit dieser Situation der Verletzlichkeit des Menschen nutzt die "Modeindustrie" sklavenähnliche Arbeit, um ihre Produktionskosten zu senken. Die Textilindustrie trägt durch die Auslagerung ihrer Tätigkeiten zu den prekären Arbeitsbedingungen bei und delegiert ihre Aktivitäten an Werkstätten mit formalen oder sogar hausgemachten Nähten.

Dieses Sklavenarbeitsausbeutungssystem, das von der Textilindustrie genutzt wird, wird als sweating system und ist die am meisten bekannte Form zeitgenössischer RC: 86627

Zugriffsverbindung: https://www.nucleodoconhecimento.com.br/sozialwissenschaften/brasilianischentextilindustrie 
Sklaverei in der städtischen Umgebung auf der ganzen Welt. Der englische Begriff, auch bekannt als "Schweißsystem" (unsere Übersetzung), bezieht sich auf Arbeitsplätze, die an ungewöhnlichen Orten durchgeführt werden, die am Ende mit Residenzen verwechselt werden und extreme Arbeitsbedingungen und miserable Löhne bieten (Cristova e Goldschmidt, 2012).

Bemerkenswert ist, dass die Hauptthemen, die von diesen Systemen geleitet werden, Ausländer sind, in der Regel aus unterentwickelten Ländern, wie einigen asiatischen und lateinamerikanischen Ländern. Denn aufgrund der Bedingung, dass sie sich in ihren Heimatländern befinden, werden sie am Ende von falschen Jobangeboten angezogen. So entsteht ein Umfeld durch den kapitalistischen Markt, in dem Unternehmen Outsourcing anstreben, um Kosten zu senken und die Wettbewerbsfähigkeit zu steigern. Der Arbeiter wird der Arbeit unter Bedingungen ausgesetzt, die dem Sklaven ähnlich sind, aus der Not heraus, indem er Raum für die Schaffung kleiner Nähwerkstätten gibt, die Ausbeutung durch Zwangsarbeit und erniedrigende Arbeit praktizieren (Palo Neto, 2008).

Die Arbeitsbedingungen, die dem arbeitsbedingungen analog zum sklaven, in dem sich die Arbeiter der Modeindustrie in Brasilien befinden, sind Teil der zeitgenössischen städtischen Sklavenarbeit, etwas seltener als die ländliche, aber es ist auch ein großes Problem.

Dieses Netz der Sklaverei im städtischen Umfeld steht in direktem Zusammenhang mit der Einwanderung von Ausländern, die hauptsächlich aus ärmeren Ländern Lateinamerikas kommen. Arbeit, die angezogen wird, um in den Fabriken der Kleidung zu arbeiten. Diese Tatsache schließt den internen Menschenhandel selbst, die aus dem Landesinneren Brasiliens, von kleinen Gemeinden in große Metropolen geleitet werden, nicht aus (Mattos, 2015).

Das Wachstum dieser Art von Kriminalität trat laut Santos (2015) hauptsächlich auf, was auf die zunehmende Bedeutung der Textilindustrie für den Inlandsmarkt des Landes in den letzten Jahrzehnten zurückzuführen ist, aufgrund der Expansion der RC: 86627

Zugriffsverbindung: https://www.nucleodoconhecimento.com.br/sozialwissenschaften/brasilianischentextilindustrie 
Mittelschicht und des Zugangs der unteren Klassen zu Kreditlinien, die den Konsum stark ankurbeln (Palo Neto, 2008). Im Jahr 2012 hat die Industrie des Landes 1,7 Millionen formelle Arbeitsplätze geschaffen, von denen 733.000.000 in der Bekleidungsindustrie konzentriert sind (Campos, 2015).

Da Brasilien eine für seine Wirtschaft relevante Textilindustrie hat, die zur Leichtigkeit der billigen Arbeitskräfte in den lateinamerikanischen Ländern (Nachbarn) und zur Notwendigkeit, angesichts externer Mercar wettbewerbsfähiger zu werden, hinzukommt, sind Ausländer zu einer leichten Beute für die Umsetzung des oben erwähnten Produktionssystems geworden, des sweating system.

Das sweating system ist eine Form des Outsourcings, die große Marken finden, um ihre Produktionskosten zu senken, während sie versuchen, sich von der Arbeitsverantwortung zu befreien.

Dieses System hat ebenso charakteristische Arbeitsplätze, die mit Wohnhäusern verwechselt werden, in denen Arbeitnehmer unter beklagenswerten Bedingungen arbeiten, unter Unterdrückung leiden, miserable Löhne erhalten, erschöpfende und prekäre Arbeitszeiten haben.

Diese Form der Arbeit, bei der es kein Minimum an Respekt vor Arbeitsgesetzen gibt, bei denen die Vergütung nur für die Produktion gilt, wird auch als "Schweißsystem" (unsere Übersetzung) bezeichnet. In Brasilien ist diese Art der Arbeit häufiger unter ausländischen Arbeitern (Cristova und Goldschmidt, 2012).

Einer der bedeutendsten Fälle der letzten Jahre ereignete sich 2011 in der Stadt Americana, an dem der "Zara"-Laden im Bundesstaat Sao Paulo beteiligt war, in dem die Arbeitsstaatsanwaltschaft 51 Personen (meist Bolivianer) entdeckte, die unter Bedingungen arbeiteten, die der Sklaverei in einer Bekleidungsfabrik, die die betreffende große Marke belieferte, vergleichbar waren. Die Arbeiter wurden anstrengenden Fahrten von bis zu 14 Stunden am Tag ausgesetzt und erhielten zwanzig Cent pro produziertem Stück (Cristova e Goldschmidt, 2012). 
Ein weiterer Fall von großer Resonanz war die Beteiligung des PernambucanasNetzwerks, das auch nach den Ermittlungen in den Jahren 2010 und 2011 keine Verhaltensanpassungsfrist bei der Staatsanwaltschaft unterzeichnen wollte und wegen Ausbeutung von Arbeitskräften verklagt wurde (Cristova e Goldschmidt, 2012).

In Brasilien wird der Kampf gegen diese Art von Praxis hauptsächlich von öffentlichen Stellen wie dem Öffentlichen Arbeitsministerium, der Bundespolizei, Bundes- und Landesregierungen und von internationalen Organisationen wie der OIT, die durch Überwachung und Bestrafung versuchen, diese Art von unmenschlicher Praxis einzudämmen, die hauptsächlich lateinamerikanische Ausländer betrifft, mit einer beträchtlichen Mehrheit aus Bolivien, in der Absicht, den schrecklichen Lebensbedingungen zu entkommen, die es gibt.

Laut Mattos (2015) sind die meisten Menschen, die in der Textilindustrie in Brasilien erniedrigenden Arbeitsbedingungen ausgesetzt sind, Bolivianer, die ihr Land aufgrund der prekären sozioökonomischen Situation, Der Korruption und des Regierungssystems verlassen, da das Land einige der schlimmsten sozialen Indikatoren in Südamerika hat.

Auf der Suche nach nur Profit stellen Unternehmen bolivianische Einwanderer ein und bezahlen sie nach Produktion, indem sie sie niedrigen Löhnen aussetzen und sie anstrengenden und erniedrigenden Reisen aussetzen, die bis zu 16 Stunden am Tag erreichen. Sie leiden auch unter Zwangsarbeit, angesichts der Einschränkung ihrer Freiheit, durch Schulden, die aus irregulären Sammlungen entstehen, oder weil sie illegal dokumentiert werden.

So erfüllt die bolivianische Belegschaft den momentanen Bedarf des Textilsektors, da es sich um Wegwerf-, Zeit- und Einzelarbeitnehmer ohne sozialen Schutz handelt, die die Lücke billiger Dienstleistungen, die von den Brasilianern abtrünnig sind, angemessen füllen. 
Brasilien hat den Kampf gegen diese Art von Praxis seit Jahrzehnten durch die Vereinigung von Regierungen und Nichtregierungsorganisationen übernommen. Und es war durch die Überwachung, dass viele Fälle von Bolivianern unter Bedingungen, die dem Sklaven ähnlich sind, entdeckt und bekämpft wurden. Einer der überraschendsten Fälle ereignete sich 2011 in der Stadt Americana, im Inneren von Sao Paulo, wo das öffentliche Arbeitsministerium 51 Menschen, von diesen 46 Bolivianern, die unter Bedingungen arbeiteten, die der Sklaverei ähnlich waren, auf erniedrigende und unmenschliche Weise in einer Werkstatt, die wiederum von einem großen Einzelhandelsgeschäft, "Zara", angeheuert worden war. Die Arbeiter arbeiteten durchschnittlich 14 Stunden am Tag und erhielten 0,20 (zwanzig Cent) pro produziertem Kleidungsstück (Cristova e Goldschmidt, 2012).

Ein weiterer Fall, der viel nationale Resonanz erlangte, war das große Einzelhandelsgeschäft "Marisa", das 48 Verstöße erhielt, um 16 Bolivianer in einer Situation zeitgenössischer Sklaverei in der Stadt Sao Paulo zu halten. Die Arbeiter wurden zu 14-Stunden-Tagesstunden unterworften und erhielten nur 247,00 R. (zweihundertsiebenundvierzig Reais). Darüber hinaus wurden dokumente auf der Website gefunden, die den Handel mit Einwanderern über die Grenze belegten (Campos, 2015).

Die Arbeiter, die nicht vor den meisten anderen Fällen in Brasilien herausgeführt wurden, waren größtenteils bolivianische Einwanderer, die durch Beschäftigungsversprechen und bessere Lebensbedingungen gelockt wurden. Bei der Ankunft in Brasilien war die Realität jedoch eine andere. Sie waren in einem Schuldensystem gefangen, das hauptsächlich Lebensmittel, Transport und Dokumentation betrafen (Mattos, 2015).

\section{SCHLUSSFOLGERUNGEN}

Durch seine neue Kleidung wurde die zeitgenössische Sklavenarbeit für einige Zeit unsichtbar, aber mit den Bemühungen internationaler Organisationen und mehrerer Länder wurde er nun anerkannt und konzeptioniert, und sein Kampf ist möglich. 
Die Faktoren, die es ermöglichen, dieses Verbrechen zu begehen, auch wenn es heute im Wesentlichen mit einem Stativ zusammenhängt: Straflosigkeit, Armut und Profit.

Die Situation des Elends der bedürftigsten Bevölkerung zwingt sie, sich unter untermenschlichen Bedingungen einer Arbeit zu unterziehen. Im Falle der Textilindustrie werden diese Arbeiter durch Nähwerkstätten genötigt, die wiederum ausgelagerte Dienstleistungen für große Einzelhandelsgeschäfte erbringen und das sweating systemder Produktion kennzeichnen.

Die Wettbewerbsfähigkeit des Marktes, die Rentabilität um jeden Preis anstrebt, führt dazu, dass der Textilsektor die Arbeitnehmer täglich sechzehn Stunden an ungesunden Orten unterstellt und ihrer Freiheit beraubt wird.

Bei diesen Textilarbeitern handelt es sich hauptsächlich um Einwanderer aus benachbarten, unterentwickelten Ländern aus Lateinamerika. Das Wichtigste Land, das Arbeitskräfte liefert, ist Bolivien, das eine große Bevölkerung in Armut hat, die bessere Lebensbedingungen im Nachbarland anstrebt.

Brasilien war eines der ersten Länder der Welt, das diese Art von Arbeit anerkannte, und dass gemeinsam mit der Internationalen Arbeitsorganisation (OIT) und austernden Nichtregierungsorganisationen versuchen, diese kriminelle Praxis auf ihrem Territorium zu bekämpfen.

Daher ist Sklavenarbeit in Brasilien und in der Welt immer noch Realität und findet Bedingungen, um sich im brasilianischen Textilsektor auszumehren.

\section{VERWEISE}

ARAÚJO JÚNIOR, F. M. Dano moral decorrente do trabalho em condição análoga à de escravo: âmbito individual e coletivo. Revista do Tribunal Superior do Trabalho, v. 72 , n. 3, p. 87-104, 2006. 
AUDI, P. A escravidão não abolida. In: VELLOSO, G. e FAVA, M. N. (Ed.). Trabalho Escravo Contemporâneo: o desafio de superar a negação. São Paulo SP: LTr, 2006.

BALES, K. Gente descartável: A Nova Escravatura na Economia Mundial. Lisboa: Editorial Caminho, 2001.

BRASIL. Lei n. 2.848, de 07 de setembro de 1940. Institui o Código Penal., Brasília DF, 1940. Disponível em: < http://www.planalto.gov.br/ccivil_03/decretolei/Del2848compilado.htm >. Acesso em: 02 de jun. 2018.

. Código penal - Decreto-lei no $2.848 / 1940$. TÉCNICAS, C. D. E. Brasília DF: Senado Federal: 138 p. p. 2017.

BRITO FILHO, J. C. M. D. Trabalho com redução do homem à condição análoga de escravo e dignidade da pessoa humana. Genesis: revista de direito do trabalho, v. 23, n. 137, p. 673-682, 2004.

CAMPOS, L. R. J. D. 0 trabalho análogo à condição de escravo no setor têxtil brasileiro. 2015. 41p. (Especialização). Universidade Tuiuti do Paraná, Curitiba PR.

COSTA, C. Para que serve a 'lista suja' do trabalho escravo? , 2015. Disponível em: $<$

https://www.bbc.com/portuguese/noticias/2015/04/150402_trabalho_escravo_entend a_cc >. Acesso em: 17 jun. 2018.

CRISTOVA, K. G.; GOLDSCHMIDT, R. O trabalho escravo contemporâneo no Brasil. Anais III Simpósio Internacional de Direito: dimensões materiais e eficaciais dos direitos fundamentais. IIISID. Chapecó SC: 24 p. 2012.

DAMIÃO, D. R. R. Situações análogas ao trabalho escravo: reflexos na ordem econômica e nos direitos fundamentais. São Paulo SP: Letras Jurídicas, 2014. 
DOU. LEI № 14.946, DE 28 DE JANEIRO DE 2013. São Paulo SP, 2013. Disponível em:

http://dobuscadireta.imprensaoficial.com.br/default.aspx?DataPublicacao=20130129 \&Caderno=DOE-I\&NumeroPagina=1 >. Acesso em: 28 maio 2018.

FAUSTO, B. História do Brasil São Paulo SP: Editora da Universidade de São Paulo, 2004

GERHARDT, T. E.; SILVEIRA, D. T. Métodos de pesquisa. Porto Alegre RS: Editora da UFRGS, 2009. 120p.

GORENDER, J. O escravismo colonial. São Paulo SP: Ática, 1985.

GRECO, R. Curso de Direito Penal: parte especial. Niterói RJ: Impetus, 2008.

LIMA, T. C. S. D.; MIOTO, R. C. T. Procedimentos metodológicos na construção do conhecimento científico: a pesquisa bibliográfica. Rev. Katál., v. 10, p. 37-45, 2007.

MARTINEZ, L. Curso de Direito do Trabalho: relações individuais, sindicais e coletivas do trabalho. São Paulo SP: Saraiva, 2015.

MARTINS, S. P. Direito do Trabalho. São Paulo SP: Atlas, 2011.

MATTOS, C. N. S. D. Análise contemporânea do trabalho análogo ao escravo na indústria têxtil. 2015. 56p. (Graduação). Centro Universitário Eurípides de Marília UNIVEM, Marília SP.

MIRANDA, C. C.; OLIVEIRA, L. J. D. Trabalho análogo ao de escravo no brasil: necessidade de efetivação das políticas públicas de valorização do trabalho humano. Revista de Direito Público, v. 5, n. 3, p. 150-170, 2010.

MONTEIRO, L. A. Políticas públicas para erradicação do trabalho escravo contemporâneo no Brasil: Um estudo sobre a dinâmica das relações entre os 
atores governamentais e não-governamentais. 2011. 184p. (Mestrado). Fundação Getulio Vargas, Rio de Janeiro RJ.

MONTENEGRO, A. T. Reinventando a liberdade: a abolição da escravatura no Brasil. São Paulo SP: Atual, 1997.

MOTA, M. B.; RAMOS, B. P. História das Cavernas ao Terceiro Milênio. São Paulo Sp: Moderna, 1999.

MTE. Quadro geral de operações de fiscalização para erradicação do trabalho escravo - SIT/SRTE - 1995/2010. Brasília DF, 2018. Disponível em: < http://www.mte.gov.br/fisca_trab/quadro_resumo_1995_2010.pdf >. Acesso em: 18 jun. 2018.

NASCIMENTO, A. M. Curso de Direito do Trabalho. São Paulo SP: Saraiva, 2010.

OLIVEIRA, J. S. D. O trabalho escravo conteporâneo no Brasil. 2011. 50p. (Especialização). Universidade Anhanguera-Uniderp, Fortaleza CE.

PALO NETO, V. Conceito jurídico e combate ao trabalho escravo contemporâneo. São Paulo SP: LTr, 2008. 128p.

PINSKY, J. Escravidão no Brasil. São Paulo SP: Contexto, 1992.

PRADO, A. A. Trabalho escravo hoje. Brasília DF, 2005. Disponível em: < https://www.anamatra.org.br/artigos/863-trabalho-escravo-hoje-09477223427479363 >. Acesso em: 15 jun. 2018.

PRONER, A. L. Neoescravismo: análise Jurídica das Relações de Trabalho. Curitiba PR: Juruá, 2010.

RODRIGUEZ, P. A. D. S. Princípios Constitucionais aplicado ao Direito do Trabalho: Colisão de Princípios nos casos concretos. 2015. 49p. (Especialização). Universidade Federal do Rio Grande do Sul, Porto Alegre RS. 
SARAIVA, R. Direito do Trabalho. São Paulo SP: Método, 2012.

SILVA, J. B. D. Trabalho escravo rural no brasil contemporâneo - uma ofensa à dignidade humana. 2009. 45p. (Especialização). Instituto Brasiliense de Direito Público, Brasília DF.

SILVA, M. R. D. Trabalho análogo ao de escravo rural no Brasil do século XXI: novos contornos de um antigo problema. 2010. 280p. (Mestrado). Universidade Federal de Goiás, Goiânia GO.

SIMÓN, S. L.; MELO, L. A. C. D. Direitos humanos fundamentais e trabalho escravo no Brasil. São Paulo SP: LTr, 2007.

SOARES, S. F. Notas estatisticas sobre a producção agricola e carestia dos generos alimenticios no Imperio do Brazil. Rio de Janeiro RJ, 1860. Disponível em: < http://www2.senado.leg.br/bdsf/handle/id/221678 >. Acesso em: 20 jun. 2018.

Eingereicht: Mai 2021.

Genehmigt: Mai 2021. 